\title{
3D laser scanning technology in the application of modeling in mining subsidence area
}

\author{
Zhao Xiaoping ${ }^{1, a}$, Zhang Jian ${ }^{2, b}$, Liu Wenlong ${ }^{1, c}$ \\ 1.Beijing Polytechnic College, Beijing, China 100042 \\ 2.Remote sensing surveying and Mapping Institute in Henan province Zhengzhou, China 450003 \\ azxpcumtb@126.com, b461910321@qq.com, clwl@bgy.org.cn
} Keywords: 3D laser scanning technology; mining subsidence; points cloud filtering ; Kriging
interpolation method.

\begin{abstract}
By means of 3 d laser scanning technology has rapidity, non-contact, high density, high precision features, such as the mining subsidence area on the station point cloud data registration, denoising, model building, such as pretreatment, can get real and effective information and visualization expression results subsidence area. The research results show that the $3 \mathrm{~d}$ laser scanning technology is applied to the mining subsidence area, to build accurate model of subsidence area, meet the requirements of subsidence analysis, has engineering practical value.

Currently, as pillar energy, coal is mined on a big scale. However, mining brings a lot of environmental and geological problems to diggings, such as subsidence, building damage and harmful substances into the ground wate ${ }^{[1]}$.Traditionally, levelling instrument and total station are used to monitor the subsidence in diggings. With the development of surveying technology, many new techniques are also applied. Sheng Yehua et al. acquired digital image pairs of subsidence area by high resolution digital camera, built DTL algorithmic model on the basis of image control points set on site, and calculated a series of 3D coordinates which can describe the morphological characters of subsidence area. Under the support of GIS, DTM of initial terrain and status of subsidence area were built. Through the analysis of two-stage DTM, specific morphological characteristic parameters of subsidence area were calculated ${ }^{[6]}$. Shi Xianling, Guo Hongxing et al. set up monitoring network and measured spatial coordinates of stations by GPS-RTK and GPS quasi dynamic measuring. After solving and analyzing the data, they found an law of surface movement with good effect ${ }^{[2-4]}$.

With the help of 3D laser scanning technology, we can effectively get dense point cloud data and large-scale subsidence information. The biggest advantage of this technology is that 3D laser scanning can acquire high-precision point cloud data directly without prism. Under the advantage of fast acquisition of high-precision 3D information, 3D laser scanning technology can collect a large number of dense point cloud data of subsidence area in a short time. And it can build DTM of complex and anomalous scence quickly, response the true appearance of subsidence basin. Moreover, this is the basis of calculating estimated parameters and analyzing ground movement. Compared with artificial measurement, 3D laser scanning technology is competitive and has more broader development space.
\end{abstract}

\section{General situation of study area}

In order to study the scheme of observation station layout of surface movement, we chose a coal mine in Jiaozuo as experimental area. Under the influence of mining, the subsidence area is calathiform and the size is $140 \mathrm{~m} \times 120 \mathrm{~m}$. This area is of wide vision, and there is no big trees and buildings. In this test, we use Leica HDS 4400 3D laser scanner, high density mode is used to collect data. Panoramic scan once requires $12 \mathrm{~min}$. If the effective scanning radius is $80 \mathrm{~m}$, the scanning area is $20096 \mathrm{~m}^{2}$. 


\section{Acquisition of point cloud data}

Acquisition of data with 3D laser scanner can divide into three parts: lay observation stations, scan and scan accurately. In the scanning phase, scanning should be carried out continuously, and the cycle of scanning should be shortened. Scanning plan should be adjusted according to actual situation. If the scanning area is flat and bare, we can increase sampling interval and shorten the scanning time. Conversely, if the terrain is complex or there is many vegetation, the sampling interval should be decreased; when scan the serious occlusion area, it is different to get point cloud data, we should add observation stations. Observation stations are designed on the maximum subsidence main section, both trend and dip section, just like traditional hatching observation stations. In the two segment of observation line, we should ensure that the scanning area must be larger than subsidence area.

\section{Point cloud data preprocessing of subsidence area}

\section{Topographic LiDAR point cloud data registration}

Registering according to points of the same name is a common method to fit point cloud data into unified coordinate system. At first, we put three or more targets in the scanning area. Scan the target area with high density mode and get the point cloud data of scanning area. After scanning the target area, we scan the targets precisely and extract the center point coordinates. The conversion parameters are calculated by using common point coordinate. When register the data among all stations, the error is less than $1 \mathrm{~cm}$. The impression drawing is showed in fig. 1 .

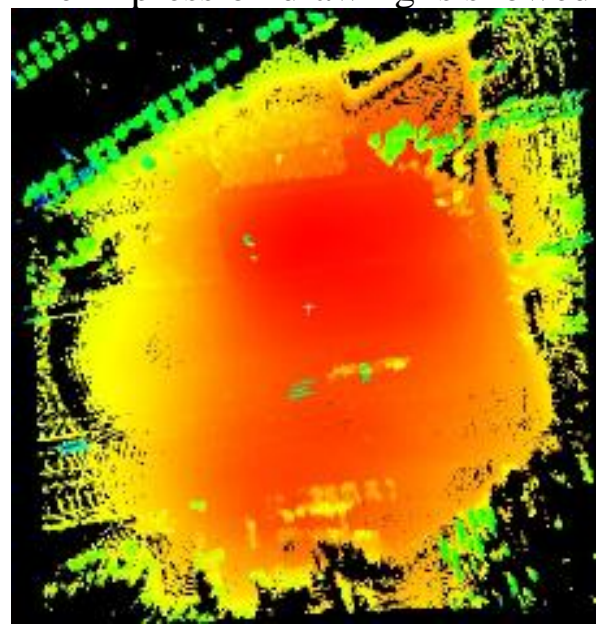

Fig 1. Splicing map of initial point cloud data of subsidence area

\section{Point cloud data denoising and optimization}

point cloud data denoising

The main task of the laser point cloud data pre-processing is how to accurately and effectively remove the chaotic noise data, retaining only the measured region exposed the land surface information. Ground 3D laser scanner in the process of data acquisition, will be recorded to all the objects can be scanned information, such as building(structure), water, vegetation and the land surface information. Different objects have different characteristics, in general, in addition to the land surface of real data, the 3D laser scanning data of subsidence area can be divided into high elevation of the building(structure) data and data of weeds in low elevation.

data optimization

Point cloud data optimization is generally divided into two kinds, removal of redundancy and sparsing simplify. Redundant data refers was given a complete point cloud model, but also generate a large number of overlapping data after multi-station data registration. This overlap of data will take up a lot of resources, reduce operating and storage efficiency, but also affect the efficiency and quality of the modeling. In some non-essential stand point cloud may appear too close, then use sparsing simplified. In the laser point cloud data of subsidence area, there are obvious geometrical features of building(structure), trees and electric poles. The average height of the building(structure) 
is the highest, and the elevation of the ground objects such as trees and poles is between the surface point and the elevation of building points. Therefore, according to the different features of different elevation to remove some noise data.

Data filtering process

According to the principle of filtering and the characteristics of subsidence area, first of all statistical data points within each elevation point cloud distribution range, and draw elevation map. According to the point cloud data elevation distribution set threshold, then remove a portion of higher elevation of the noise, then, after the removal of data in accordance with X-Y plane grid. The study area is divided into $\mathrm{M} \times \mathrm{N}$ regular grid, and then import the split data into software for processing, only preserve the surface data, remove the low vegetation information of the point cloud.

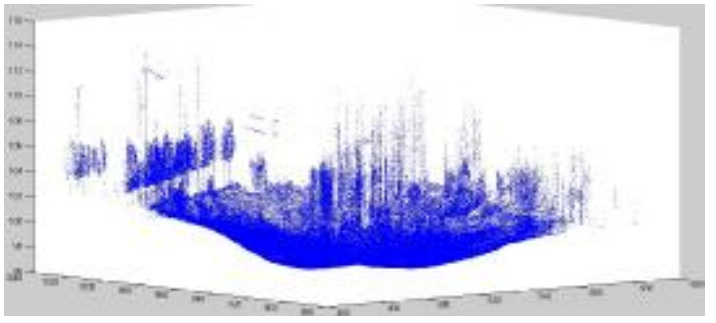

(a) Noise data before removing

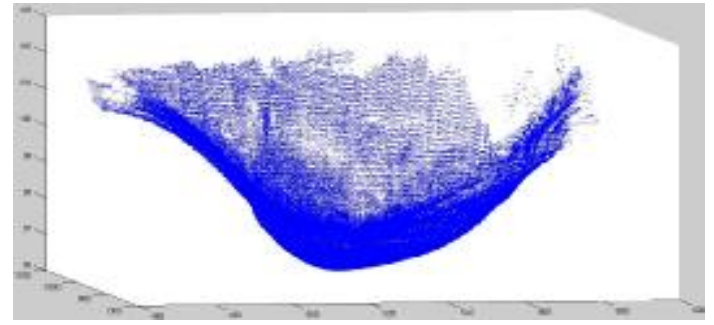

(b)Noise data after removing

Fig.2 Comparison of point cloud before and after denoising

As can be seen from figure 2(a), the subsidence zone is large, rough filtering can not be completely removed some low noise, and it is possible to put a higher surface elevation point mistook the noise data to removed. In order to solve this situation, the situation is complicated areas must be divided into a plurality of fine grids. Filter out the noise within each grid, After the grid division, it can be further removal of these low vegetation in the small grid,as can be seen from figure 2(b).But this method cannot automatically and completely filter the noise of low vegetation, to be further improved.

\section{Build the surface model}

For non-topological relations point cloud data, the model poorly constructed using these scattered points directly. To get a complete point cloud data, and accurate expression of surface subsidence characteristics, needs to use the interpolation method to compensate for the missing data. There is a lot of interpolation, Kriging interpolation method is a commonly used interpolation method based on geostatistics, the method can be used to generate a prediction surface, but also given the accuracy of the results or the measure of certainty. After the data interpolation process, it need to build a grid model of the scanning area, it is not only able to clearly express the basic information of terrain surface, but also preparing for the forecast and analysis of future changes in the terrain surface. According to the characteristics of subsidence area, choose Kriging interpolation to fill the voids, by adjusting the spacing of the required grid, such as figure 3 . According to different elevation, giving different colors and eventually generate terrain surface renderings, such as figure 4 .

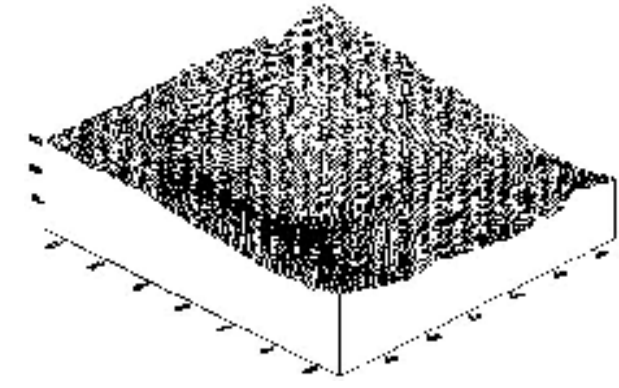

Fig. 3 Grid map

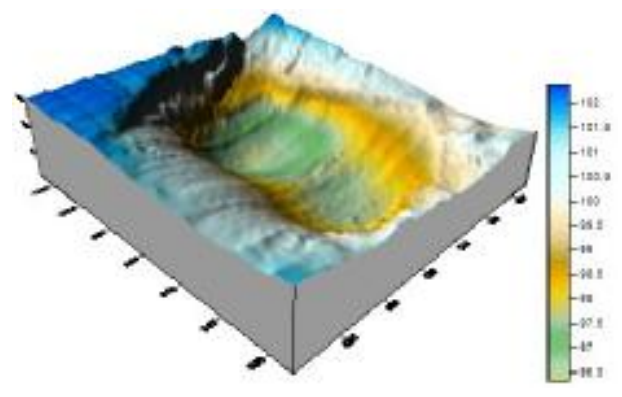

Fig. 4 The surface

rendering model 


\section{Comparison of section line point cloud data}

When using 3D laser scanner to set observation station, it can be set up along the lines ends to higher places. This can not only guarantee the point cloud density in the central area of the subsidence basin, but also can give attention to the accuracy of the point cloud in the edge region of the subsidence basin.

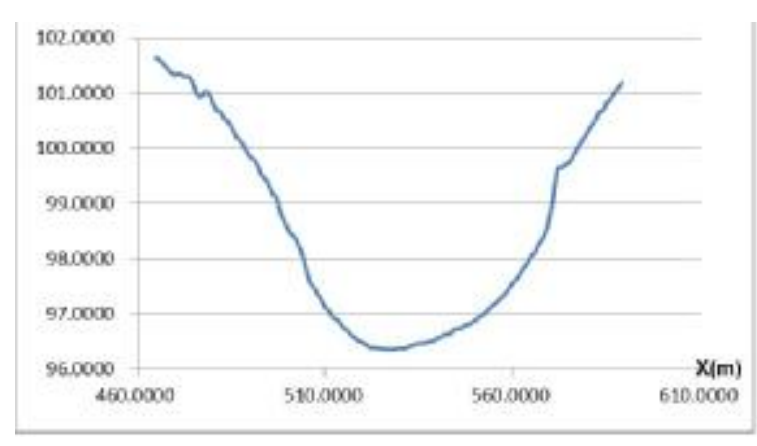

) dip section diagram

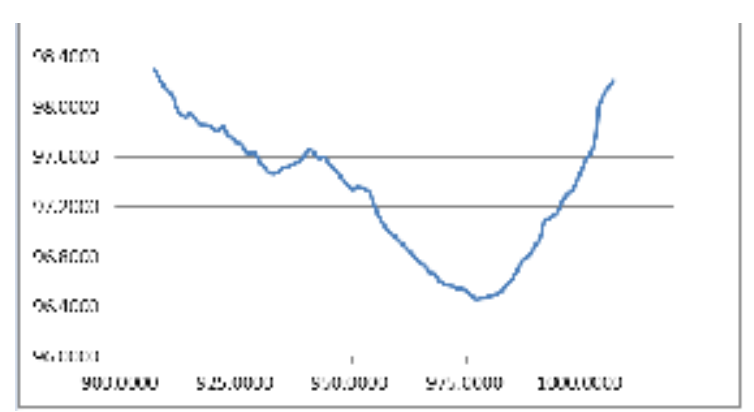

(b) trend section diagram

Figure 5 The subsidence curve profile diagram

The data points collected by 3D laser scanner, can make the seam trend(or dip section) of the analysis of the line based on the 3D modeling, more accurate than the traditional measurement methods to reflect the trend of surface changes. When analyzing the subsidence, as shown in the figure 5 of the sinking value, it can be obtained by the subtraction value of the data before and after deformation.

\section{Concluding remarks}

This paper is on the basis of analyzing the ground 3D laser scanner for mining subsidence monitoring, through the method of stepwise refinement grid, it can effectively remove the point cloud noise, provide basic data for application of subsequent point cloud. For the filtered produce "empty" point cloud data, Kriging interpolation method is used to interpolate the data, obtained better effect surface model. Compared with traditional research methods, 3D laser scanning technology has great advantages in 3D modeling and deformation analysis of mining subsidence area.

\section{References}

[1] Kan Wu, Yunjia Wang, Suiquan Wang, mining subsidence monitoring and forecasting technologies [M], the Chinese environmental science press ,2012.

[2] Xianling Shi, Shubi Zhang. GPS application to dynamic measurement of land surface movement observation station [J]. Western exploration engineering,2006,(12):137.137.

[3] Gang Wang,Guangli Guo,Ling Li. GPS-RTK application of coal mine surveying and mapping and surveying in the mountains [J]. China mining and construction,2011,40 (2): 41.

[4] Hongxing Guo, Yufu Hang, Baoji Zhao. RTK technology and its application in mining subsidence monitoring [J]. Shanxi coal science and technology,2007 (2): 31-33.

[5] Jingjing Li,Zengzhang Guo. Based on D-InSAR technology of mining subsidence in coal mining area [J]. Journal of Henan Polytechnic University (natural science Edition),2006,25 (4): 306-309.

[6] Yan Jiang,Junhai Gao. Synthetic aperture radar interferometry technology application to mining subsidence monitoring [J]. Mine survey,2003 (1): 5-7.

[7] Wensheng Mei, Zhenglu Zhang, Quanyi Zhang. Study on the application of survey robot in deformation monitoring [J]. Dam safety, 2005(5):33-35.

[8] Zhenli Ma, Changdong Ji, Dongfeng Ren. GPS joint operations with the robot in deformation monitoring of open pit applications [j]. Mine surveying, 2007(1):41-42. 
[10] Shuanghong Wang, Bin Wang. Application of survey robot in deformation monitoring [J]. Journal of disaster prevention technology College, 2005 , 7(3):38-42.

Project funding on the market in Beijing, Project No.: KM201610853006.

Author introduction: Zhao Xiaoping(1976-),female, Han, Xinzhou Shanxi Province, associate professor, major in teaching and studying new technology of surveying and mapping. 